\title{
La pratique autobiographique des cheminots dans le fonds de l'APA
}

Autobiographical writing in the APA (Association for Autobiography collection)

Véronique Leroux-Hugon

\section{OpenEdition}

\section{Journals}

Édition électronique

URL : https://journals.openedition.org/rhcf/1634

DOI : 10.4000/rhcf.1634

\section{Éditeur}

Rails \& histoire

Édition imprimée

Date de publication : 10 février 2013

Pagination : 103-111

ISSN : 0996-9403

Référence électronique

Véronique Leroux-Hugon, "La pratique autobiographique des cheminots dans le fonds de l'APA », Revue d'histoire des chemins de fer [En ligne], 44 | 2013, mis en ligne le 13 novembre 2014, consulté le 22 avril 2022. URL : http://journals.openedition.org/rhcf/1634 ; DOl : https://doi.org/10.4000/rhcf. 1634 
Véronique LEROUX-HUGON

\section{La pratique autobiographique des cheminots dans le fonds de l'APA}

on propos vise à illustrer la présentation de l'APA par quelques exemples puisés dans ce fonds particulier. Il y a deux raisons pour évoquer ces exemples : nous sommes très fiers de la plaque apposée à l'entrée d'Ambérieu, la désignant comme la ville de l'autobiographie, et puis Ambérieu « la cheminote " (comme l'appelle Robert Vannet) est, nul ne l'ignore, un nœud ferroviaire, belle métaphore que nous reprendrons pour imaginer tous ces croisements de vies qu'on rencontre au fil des lectures autobiographiques.

Je voudrais esquisser une brève typologie des textes, noter les circonstances et les pratiques d'écritures et, dans un second temps, rappeler le déroulement de ces vies au service, continu ou pas, du chemin de fer, telles que les cheminots au sens large les ont racontées.

\section{Typologie}

Quelques chiffres d'abord, pour ne pas y revenir : la recherche dans le Garde-mémoire et dans la base de données de l'APA a été menée sur une vingtaine d'années (1992-2010) avec les termes suivants : "chemin de fer ", "SNCF ", " cheminot " et " train » (ce dernier terme ne se révélant pas significatif). Rappelons que la collecte des textes déposés à l'APA est tout sauf 
systématique, compte tenu des ambitions de l'association, et sa variété est l'un des charmes du fonds. Il en est résulté une cinquantaine d'occurrences qu'on pourrait trier grossièrement en deux catégories : les personnes ayant travaillé aux chemins de fer (19 textes) et leurs descendants (enfants, conjoints le plus souvent) quand ils les évoquent, ce qui est parfois l'essentiel de leur texte (une vingtaine). J'ai opté pour une lecture intégrale des textes, de taille variable, lecture complétée par celle des Échos-du-Je, ces fameux comptes rendus qui fournissent une lecture objective et plurielle des manuscrits.

D'emblée, des restrictions et la première est d'importance car j'ai triché sur l'enjeu : ces textes ne sont pas exclusivement écrits par des cheminots ou sur les cheminots, $j$ 'ai retenu volontairement plusieurs auteurs qui travaillaient dans l'administration, dans les bureaux d'ingénieurs, évoquant cet univers très spécifique du train. Ainsi, le Livret 4 de Pierre Marbotte, une entreprise intéressante de correspondances croisées dédiée aux petits-enfants, traite de la construction des chemins de fer indochinois en 1903 : une touche d'exotisme dans un paysage essentiellement européen.

Autre limite, je ne me suis pas attardée sur les textes évoquant le passage aux chemins de fer de manière très rapide et sans que ce passage laisse une trace marquante dans le récit de vie.

Sans surprise, les auteurs sont masculins en majorité. Ainsi, c'est pour rappeler les Souvenirs de mon père que Marie Capron dresse son portrait de cheminot. Autre constatation, signalée plus haut, Ambérieu et le Bugey sont amplement mis en scène par Robert Vannet, Fernand Piralla, Georges Martin pour ne citer que ceux-là. Il serait amusant de dresser une carte des voies telle que celle affichée dans les compartiments de train et de pointer les textes correspondants, déposés à La Grenette, temple de la pratique autobiographique...

Quel est l'âge du chef de gare ? Les textes évoquent la fin du XIX ${ }^{\mathrm{e}}$ siècle, celui de Paul Brustis né en 1877 et surtout le $\mathrm{Xx}^{\mathrm{e}}$, la majorité des déposants étant nés entre 1900 et 1960. Jean Simon, cheminot et militant sétois, se souvient de mai 1968, Françoise Bonnot reconstitue la trame des sixties. Le "grand chantier " du XXi ${ }^{\text {e }}$ siècle (que d'aucuns surnomment le grand chambardement), pour reprendre le titre d'un article du Monde d'avril 2011 ${ }^{1}$, n'étant, on le comprend bien, pas évoqué.

Par pratiques autobiographiques, il est temps de préciser que l'on entend ici celle des journaux intimes (tel celui de Jean Simon justement, ou de Françoise Bonnot-Jörgens) et celle des récits de vie, consacrés au travail dans sa totalité, ou a contrario à un seul épisode marquant. De plus, pour Georges

1- Benoît Hopquin, «SNCF, le grand chantier », Le Monde, 10 avril 2011. 
Creux ou Robert Vannet par exemple, la carrière ne se déroule pas toujours entièrement aux chemins de fer, auxquels seront donc consacrés de manière variable quelques pages, la totalité du texte ou une partie importante.

Cette nuance nous amène immédiatement à nous interroger sur les raisons de cette pratique d'écriture chez les cheminots et à proposer une hypothèse qu'on va essayer d'argumenter. Il me semble en effet que c'est la conscience très forte de la possession d'un savoir-faire, d'une compétence professionnelle solide, le sentiment aussi de vivre une aventure exceptionnelle (le terme revient à plusieurs reprises) comme acteurs principaux dans les différentes compagnies devenues SNCF dans la foulée du Front populaire qui les a incités à écrire ou à rassembler les souvenirs. Au même titre, c'est sous l'impression d'une image forte que les proches de cheminots ont évoqué ces figures quasi héroïques, dans l'imaginaire enfantin tout au moins. Parfois on saute une génération et ce sont les petites-filles qui recueillent et transcrivent avec des moyens modernes les souvenirs des grands-pères (tels Jean Pons ou André Dismier) ou entreprennent de reconstruire la saga familiale, comme Alain Pignon ou Pierre Marbotte. Plus original, dans O farol (le Phare), Carlos Rato, fils du militant Tomaz, prévient que ce livre est " une synthèse de mémoires, la mienne, celle de Tomaz et celle de ses compagnons galériens ".

Dans bien des textes déposés, et c'est souvent le cas pour les cheminots, l'auteur explique d'emblée ce qui l'a poussé à écrire : soit immédiatement, comme Gabriel Sylvestre, soit plus souvent avec le recul et la disponibilité de la retraite, recul qui ternit parfois la fraîcheur du souvenir mais autorise le bilan et la vision d'ensemble.

"Voici, exhumé d'un fond d'armoire où il jaunissait depuis près de 20 ans, un fragment de mémoire [...] [Comme témoin] je me contente de classer tracts, télégrammes, articles, de mettre en forme des notes prises au jour le jour..." (Jean Simon).

André Dismier, rédigeant à 70 ans, Georges Creux, racontant à 85 ans ses "Aventures et mésaventures " ou Robert Vannet, entreprenant à 71 ans de rédiger enfin ses " histoires ", tous prennent eux-mêmes la plume, commencent par raconter dans le désordre, s’organisent enfin : "Écris ta page et raconte nos bons et mauvais souvenirs qui ont défilé bon train au chemin de fer $[s i c]$ " conseille Vannet à un copain, après s'être lui-même lancé sur des bouts de papier.

Paul Brustis, usé par 65 années bien pesées comme cheminot, décide à la fin de sa vie de "transmettre " et décrit sa vie dans deux manuscrits dont le second s'intitule "Ma carrière, toute ma vie ", tandis que Pierre Cottereau intitule le sien " Pour que la mémoire ne meure ». 
Nécessité de témoignage donc, de transmission, de révérence aussi face aux souvenirs des aînés, la démarche est relativement banale. Une autre urgence impose l'écriture immédiate, celle du traumatisme individuel (l'accident par exemple) ou collectif, je fais référence aux conflits sociaux, aux deux guerres, puisque le texte de Maurice Lemaire s'intitule " Journal des deux guerres ». L'écriture en est alors différente, plus hachée et spontanée comme celle de Gabriel Sylvestre. Dans ce dernier cas de figure, l'usage du « je » et du présent s'imposent, au contraire des souvenirs rédigés au passé composé ou à l'imparfait.

Enfin, j'y reviendrai, les textes sont rédigés dans une langue simple, avec souvent des bonheurs d'écriture et le recours à des termes techniques que leurs utilisateurs s'efforcent toujours de définir et d'expliciter : le monde des roulants et du PLM a son vocabulaire dont il est fier.

\section{Une vie aux chemins de fer?}

Par rapport au déroulé habituel d'un récit de vie, je vais tenter de tracer celui du " cheminot-déposant ", néologisme fort maladroit pour signaler que l'ensemble des exemples cités est choisi exclusivement parmi les écrits confiés à l'APA. Quelle est la vie quotidienne, comment sont vécues et transmises les ruptures - dans quel contexte économique et social (pour évoquer un monde sans doute en voie de disparition) - à travers ces « écritures ordinaires du chemin de fer ", pour paraphraser le titre d'un colloque ${ }^{2}$ organisé en Sorbonne par l'AHICF en 1996 ?

Tout d'abord pourquoi et comment y entre-t-on ? C'est souvent l'exemple des pères et des grands-pères, les uns gardes-barrières sémaphoristes, les autres ajusteurs, poseurs de voie, car être cheminot de père en fils est une réalité et aussi une garantie de l'emploi : "Tu devrais faire une demande, c'est intéressant, ça serait une place sûre si tu y arrives, c'est comme si tu étais fonctionnaire, il y a la retraite [...]", propose son beau-père à Georges Creux. Plus tôt, Paul Brustis se trouvant " assez discipliné " opte en 1903 pour les chemins de fer et a recours à la recommandation de son capitaine. Dans les mêmes années, André Dismier, fils de " tout petit employé ", est présenté par son père. Eugénie se marie avec Chèvre sur le conseil de sa mère : "Il est aux chemins de fer, c'est un emploi sûr, tu n'auras pas de souci ». Le mariage sera malheureux, Eugénie ne s'habituant pas à la vie de son roulant de mari, mais c'est une autre histoire. Les horreurs de la Première Guerre incitent également

2- Voir François Moureau, Marie-Noëlle Polino (dir.), Écritures du chemin de fer, Paris, Klincksieck, 1997. 
les rescapés, quelquefois blessés, à postuler à un emploi : ainsi Paul Poncelet, blessé en 1916, note qu'en 1918 il a "opté finalement pour le chemin de fer en raison d'une obsession subie par beaucoup de ceux qui sortaient du cauchemar de cette guerre interminable : il s'agissait de choisir une profession vous mettant à l'abri de tout retour sur un front de guerre; le chemin de fer, où l'on était mobilisé dans son emploi, donnait cette garantie... ». Jean Pons, libéré des obligations militaires et petit-fils de gardes-barrières, trouve facilement à s'embaucher comme pointeur-releveur au PLM, après un examen écrit.

On entre dans un petit emploi, comme aide, avec une promotion plus ou moins rapide selon que le supérieur vous est hostile ou favorable et qu'on montre son désir et ses aptitudes à suivre des cours et à se perfectionner : c'est le cas de Robert Vannet, tombant dès son premier jour sur un chef acariâtre, le "Ronnu ", bien décidé ensuite à passer l'examen de facteur aux écritures. Pierre Cottereau ou Paul Brustis décrivent bien la lente mais réelle progression d'une longue carrière : ce dernier débute comme homme d'équipe, il termine chef de gare hors classe; dans un autre contexte Fernand Piralla évoque son parcours : d' " auxiliaire mineur ouvrier ajusteur", il devient chauffeur de route, mécanicien, conducteur électricien.

La gestion des différentes compagnies ou de la SNCF exige une administration considérable qui doit veiller à tout, ce qui implique le travail indispensable dans « les bureaux » qui sont parfois le premier stade, voire le seul, d'une carrière. Les acteurs n'en sont pas ici les cheminots du terrain, je l'ai dit, mais les témoins à de multiples échelons : inspecteurs, contrôleurs, ingénieurs de l'univers ferroviaire.

\section{Courteline aux chemins de fer}

Avec des descriptions savoureuses, Paul Couget raconte son embauche expéditive au côté de spécialistes $\mathrm{du}$ " raccordement parabolique » au « Bureau d'Études de la voie " à Tours, où l'ambiance chahuteuse compte bien plus que le temps consacré à lever des profils. On retrouve le même humour chez André Dismier : ce dernier, revêtu d'un beau complet neuf, est tout fier d'être embauché en 1906 pour un travail dans les bureaux centraux, où " régnait alors (contrairement aux gares) ce que l'on a appelé le paternalisme d'une part et le "rond-de-cuirisme" d'autre part » : chefs " coulants ", méthodes de travail antiques, absences tolérées et feuilles de présence évanescentes, arbitraire enfin des mutations et des avancements. Quand il compare ces conditions de travail avec celles de son père, en service actif à la gare de Paris-la Villette en 1880, il souligne le contraste : salaires très bas, omnipotence des chefs, journées de douze heures. 
Comme le note Micheline Lucas à propos de sa mère : "Bien sûr, on embauchait aussi des femmes au chemin de fer. " Nous sommes en 1923, sautons un demi-siècle pour un dernier exemple, celui de Françoise BonnotJörgens, pour laquelle l'année passée à la SNCF, en conciliant travail et études, sera un tremplin. En 1963, elle est affectée au $4^{\mathrm{e}}$ bureau de la rue de Saussure, au service Redevances. Elle y découvre ce monde des bureaux très particulier où les femmes sont majoritaires, plus âgées qu'elle et la prennent en affection, les conditions et le salaire médiocres, pour un travail sans grand intérêt, ce qui n'altère pas son esprit d'observation et son talent à recréer une ambiance pittoresque d'où émergent, parmi ces blouses grises, des figures caricaturales.

\section{Les roulants}

On passe évidemment à un autre monde quand on entre dans celui des roulants. Au langage de la dérision, parfois, se substitue celui de la passion pour le boulot, même si Fernand Piralla, par exemple, dit entrer par nécessité et non par vocation "dans le monde fascinant de la traction vapeur au dépôt d'Ambérieu ». Il a confié ses souvenirs à la Revue d'histoire des chemins de fer en 1993, dans un article déposé ultérieurement à l'APA.

Pour reconstituer la carrière de son père, Pierre Cottereau s'efforce de définir très exactement en quoi consistent les tâches du serre-frein, du conducteur, dont la mission ne consiste pas à conduire un train, celle-ci étant réservée au mécanicien et au chauffeur - la " caste noble des cheminots " - mais varie avec la nature du train. Et on ne mélangera point le service traction et le service exploitation. Son père va devenir enfin chef de train (avec uniforme) et maitre à bord, guidé par sa seule feuille de route. Outre l'importance de la besogne assignée à chacun, le fils excelle à évoquer l'ambiance de ces voyages, les difficultés imprévisibles et la nécessaire attention de tous les instants. Les autres témoignages évoquent pareillement un travail difficile, où la négligence ne pardonne pas, les responsabilités lourdes vis-à-vis des voyageurs et du matériel. La pénibilité est aggravée par les trois-huit et l'alternance de service dit " régulier " et de service dit de "mise à disposition ", où des " éveilleurs " doivent prévenir l'agent qui fait rarement nuit complète. Au même titre, Paul Brustis ne connaît jamais d'horaires réguliers puisqu'il est chargé de remplacer tous les chefs de gares en congé : "En conséquence, je ne couchais à la maison qu'en fin de mois, lorsque toutes les gares avaient été remplacées. » Et si tous insistent sur la hiérarchie très minutieuse des différentes tâches, c'est aussi pour souligner qu'il s'agit avant tout d'un travail d'équipe où chacun est l'engrenage indispensable pour le bon fonctionnement de l'ensemble, tout au moins à l'ère des locomotives à vapeur. Dernier stade de sa carrière, c'est 
quand il devient chef de train principal que le père de Cottereau... quitte le monde des roulants.

" Je raconte une anecdote pour mieux faire comprendre " écrit Robert Vannet, qui fait revivre d'une plume légère mais précise les mille anecdotes des 32 ans passés en trois-huit : bizutage des débuts, tant qu'il est " au sabot ", wagonnier, entre Modane, Chambéry, Bellegarde, sur le "Chou-Fleur », manipulant les pétards en cas de danger, ou narrant sa nuit la plus longue en novembre 1947.

Comme d'autres aux trois-huit, il a du temps libre qu'il sait mettre à profit, avantage secondaire auquel on peut ajouter, pour les cheminots et leur famille, la gratuité des transports, le logement de fonction, et quelques autres agréments que nos témoins notent également et qui adoucissent à peine une vie dure, aux salaires encore faibles. Les luttes syndicales évoquées ne connaissent vraiment quelques résultats qu'après la Première Guerre mondiale.

Jérôme Berlan est né dans la Bresse, en 1924 son père travaillait au PLM, sa mère était femme au foyer : "Les salaires étaient bas, les fins de mois difficiles. » Le rythme quotidien est trop souvent rompu par l'accident, accident auquel aucun des cheminots cités n'échappe et qu'il raconte, souvent longtemps après, avec la même émotion. Paul Brustis connaît sa première tuile en 1910, la perte de 19 barils de vin pour un problème d'aiguillage : contusions pour le chef de train et désespoir du malheureux, rendu responsable et classé facteur par mesure disciplinaire. Le jeune Georges Martin, issu d'une famille d'ouvriers cheminots, échappe de peu à l'accident en manquant d'être écrasé par un train de marchandises circulant tous feux éteints comme c'est la règle pendant l'Occupation. La grand-mère de Jean Pons n'ayant pas fermé la barrière à temps, un cheval s'est avancé sur la voie : "Elle avait aussitôt pris ses pétards, la lanterne et le drapeau rouge et avait couru sur les voies jusqu'au pont de la Durance pour arrêter l'Express. "

On évoque aussi à mi-voix les ravages de l'alcoolisme, très fréquent, qui atténue la vigilance, celle du père de Micheline Lucas, par exemple.

\section{Ruptures}

J'ai appelé, peut-être abusivement, ruptures les traces laissées dans l'écriture des cheminots par les conflits du Xx $x^{e}$ siècle. Cependant, si on n'en lit peu de relations directes dans les textes, on sait le monde cheminot éminemment solidaire, très impliqué dans le syndicalisme. Je n'ai pas trouvé dans le fonds, sauf brèves allusions, de récit spécifique de luttes syndicales. 
Par contre, c'est notamment à l'occasion de la Seconde Guerre mondiale que des hommes comme Georges Creux, Gabriel Sylvestre ou Georges Martin vont écrire quelques pages relatant des faits d'évasion, de résistance, parfois consacrées exclusivement à ces événements.

Georges Creux est rapidement fait prisonnier en juin 1940 et expédié dans un Stalag près de Strasbourg mais il est libéré en septembre, après avoir attendu longtemps la signature libératrice, pour rejoindre Saintes. En tant que cheminot, il travaille sous surveillance allemande dans les ateliers : travaux divers, on récupère tout ce qui est possible, car il faut aussi penser à nourrir la famille, bientôt sous la menace des bombardements américains ; dernier pointage le 7 juillet 1944, mais dangers de la guerre et difficultés quotidiennes ne cessent pas pour autant, comme il le raconte.

C'est encore Gabriel Sylvestre qui s'évade de Bavière, caché dans un wagon postal, grâce à la complicité des cheminots : de retour à Béziers en 1942, il n'en a pas fini avec l'occupant et, dénoncé pour évasion en 1943, il passera 50 jours en prison. Son texte est entièrement consacré à cet épisode crucial de sa vie, raconté au jour le jour.

Également prisonnier en Bavière, près de Nuremberg, Jean Pons déplore la longue attente de sa libération, alors que « tous les cheminots étaient rentrés en France $»^{3}$. De retour en Avignon, il est mécanicien dans les ateliers et travaille, lui, sous la menace constante des bombardements. D'autres rappellent que c'est en tant que cheminots, profession stratégique, qu'ils sont les premiers libérés, quand ils étaient P.G., pour accomplir leur travail dans les chemins de fer français.

Enfin je n'évoquerai que très rapidement la résistance des cheminots, à laquelle a été consacré en 2006 un numéro de la Revue d'histoire des chemins de fer ${ }^{4}$. Question lourde de controverses récentes, finement analysées par Christian Chevandier. Je souhaite seulement en citer deux exemples.

D'abord, le refus "intégral " du pétainisme de la famille de cheminots de Georges Martin, comme il le rappelle dans Ma résistance. Il a 14 ans en 1940, après s'être engagé dans une section-couverture des Éclaireurs de France, il participe à Ambérieu, en 1944, à un groupe de sabotage dont la mission est d'empêcher par tous les moyens la circulation des trains. Il raconte de manière très vivante le quotidien difficile dans les maquis de Dorvan et de Corlier, proches de la ville.

3- Seule la moitié des cheminots prisonniers est libérée en 1940 et 1941 [N.d.l.R]. 4- « Les cheminots dans la Résistance. Une histoire en évolution », RHCF, n 34 (2006). 
Ensuite, le président national de Résistance-fer, Maurice Lemaire, né en 1902, dont la fille a déposé le Journal des deux guerres dans lequel il évoque au jour le jour ses activités clandestines dans le Nord. Très rapidement, juste après la Débâcle, il organise des évasions de soldats anglais, puis ce sont des opérations de sabotage et il consacre plusieurs pages aux actions menées en août 1944, racontées avec un grand luxe de détails - et au présent.

De cette démarche autobiographique, on pourrait citer bien d'autres exemples, comme celui de F. Piralla, pour boucler la boucle. Dans ses Souvenirs d'Ambérieu, il a écrit une vivante synthèse de l'évolution de cet univers, sans négliger, dans cette évocation personnelle, de recourir à la précision technique avec la vivacité qui émaille son récit. Dans ce bilan, publié par la revue citée plus haut, puis confié ultérieurement à la toute jeune APA, Fernand Piralla ne propose pas une vision uniformément rose, on y décèle même une pointe d'amertume, réaliste. Le parcours s'arrête ici, les transformations considérables qu'a connues le monde ferroviaire donneront certainement lieu à de nouveaux récits.

\section{Références Bibliographiques}

Hopquin Benoît, "SNCF, le grand chantier ", Le Monde, 10 avril 2011.

Moureau François, Polıno Marie-Noëlle (dir.), Écritures du chemin de fer, Paris, Klincksieck, 1997.

Coll., «Les cheminots dans la Résistance. Une histoire en évolution", Revue d'histoire des chemins de fer, $\mathrm{n}^{\circ} 34$ (2006). 
